\title{
LA EXPRESION DE LA CONDICIONALIDAD EN ESPAÑOL
}

\author{
(CONJUNCIONISS Y LOCUCIONES CONJUNTIVAS)
}

No son muchos los trabajos dedicados al estudio de la expresión de la condicionalidad en español. El más completo, desde el punto de vista diacrónico, a pesar de su antigüedad, es el de Gessner, Die hypothelische Periode in Spanischen in ihrer Entwiliclung. y desde el punto de vista sincrónico, el de Lidia Contreras, Las oraciones condicionales. Otros trabajos de enfoque preferentemente diacrónico son los de Garcla de Diego, La uniformación ritmica en las oraciones condicionales, y el de Emilio Náñez, Sobre oraciones condicionales. El de Kany, More about conditions expressed by Spanish de plus infinitive es de tipo sincrónico. El último estudio aparecido sobre las oraciones condicionales en español, Henry Mendeloff, The Evolution of the Conditional Sentence Contrary to Fact in Old Spanish, es de tipo diacrónico y abarca un período de tiempo que va desde el siglo $\mathrm{XII}$ al $\mathrm{Xr}^{1}$.

De estos trabajos monográficos, sólo los de Gessner y Lidia Contreras se ocupan de la estructura de la oración condicional tanto en lo que a modos, tiempos y elementos conjuntivos, como a su significación

1 E. GESSNER. Die hypothetische Periode im Spanischen in ihrer Entwikelung, ZRPh, 1890, XIV, pp. 21-65; E. KANY. More about conditions expressed by Spanish 'de' plus infinitive. Hispania, 1939, XXIT, pp. 165-170; GARCí DE DIEGO. La uniformación ritmica en las oraciones condicionales, EDMP. III, IIa. drid, 1952, pp. 95-107; EM山1o NAĩ̃z. Sobre oraciones condicionales. Anales cervantinos, 1953. III, pp. 353-360; LIDIA CONTRERAS. Las oraciones condicionales, BFUCh, 1963, XV, pp. 33-109; HENRY MENDELofF. The Evolution of Conditional Sentence Contrary to Fact in Old Spanish. Washington, D. C., r960. Trabajo de sintesis y de oricntación fundamentalmente sincrónica es la tesis doctoral, inédita, presentada en la Iiacultad de Letras de la Universidad de Granada, de JosÉ PoLO l’or.o. Introducción al esludio de las oraciones condirionales en español (Ensayo de teoria gramatical). 
y límites dentro del período hipotético, se refiere. El artículo de Kany sobre de + inf. con valor condicional es el segundo que escribe el autor referido a este tipo de construcción. Como el primero, tiene un fundamento estadístico, lo que le permite asegurar que aconditional de + inf. is considerably more common today than conditional $a+$ inf. (although, the opposite was true in the Golden Age)" (p. I05). Este segundo artfculo está motivado por la aparición, en I937, de la Spanish Syntax List, de Keniston, en la que se llegaba a una conclusión diametralmente opuesta a la suya: "Great was my surprise, therefore, to discover that Keniston's relative frequency count for conditional $d e$ and $a+$ inf. was far being in accord with my own findings" (p. I65). La argumentacion de Kany y la crítica del método empleado por Keniston son absolutamente convincentes.

H. Mendeloff realiza su espléndido estudio porque el problema de la evolución de la condicional en español siempre ha sido tratado de manera tangencial y porque "the only study of appreciable dimensions which deals directly whith the problem from serious errors of omission and conission» (p. V.). Su propósito es estudiar la evolución de las oraciones condicionales irreales en español antiguo «by analyzing the most representative texts of the twelfth through the fifteenth centuryi (p. V.). En el capitulo primero se pasa revista a todo lo que se ha dicho de importante sobre la estructura de la oración condicional y sobre los factores - afectivos, lógicos, temporales- que la integran. Al final de este capítulo vuelve a exponer, pero ahora como conclusión de un largo camino, la razón de ser de su estudio: "Our survey of the work... leads us to conclude that the problem is largely unexplored, and that the need for further investigation is imperative" (p. Ig). En el capitulo segundo, Naturaleza de la investigación, que es la explanación científica de su propósito y de los puntos y apartados ya anunciados en el Preface, dice el autor algo unuy importante por lo que respecta a la materia específica de nuestro estudio: sólo tomará en cuenta aquellas construcciones irreales que presenten la prótasis y la apódosis, pero uwhose protases are formally introduced by sil (p. 24). Esta es la razón por la que haremos sólo escasas referencias a esta monografía: nuestro propósito es estudiar, precisamente, las conjunciones y locuciones conjuntivas que introducen el periodo condicional en español y que no sean si ni las que tengan si entre los elementos que las forman. En el capítulo de resultados (III) se expone la frecuencia de fórmulas de correlación temporal a lo largo de cada siglo. Un capítulo de conclusiones y un apéndice, donde se recogen los ejemplos sacados de cada obra examinada, cierran un estudio tan bueno como breve. 
Eil de García de Diego, sugestivo y claro, trata de demostrar cómo la uniformación temporal en los dos miembros de la oración condicional obedece a una ley de simetría rítmica: "Las condicionales de las diversas lenguas se rigen por la ley lógica de los tiempos, hasta que, oscurecido el concepto preciso del tiempo, se impone la simetría rítmica de los verbos, ya por generalización de un caso particular lógico en que se da esta simetría, ya por ciega tendencia de simetrian' ${ }^{2}$. La uniformación temporal de origen vulgar que responde a la fórmula 'si tendría, daría' puede ser incorrecta desde el punto de vista normativo, pero, al igual que las correspondencias temporales que se aceptan como típicas y que se consideran correctas dentro del marco de la consecutio temporum de la expresión del periodo hipotético condicional en español obedecen a una ley genética, también esta fórmula tiene la suya, lingüísticamente justificable y explicable. $\mathrm{Y}$ "es pueril la objeción de los gramáticos de que la fórmula 'si j'aurais, je donnerais' es incorrecta, porque, si no responde a la ley de lógica temporal, responde a la ley sensorial y estética, que ha usado los tipos modelos del latín literario y del castellano de oro... Iis pueril para el lingüista, aunque no lo sea para el purista, el censurar 'si tendría, daría', porque lo dice el vulgo, y el aprobar 'si pudiera, lo hiciera', porque lo decía Cervantes, pues esta última fórmula, en su desarrollo, llegó a ser lógicamente impropia» "2. Está, pues, concebido este trabajo diacrónicamente, pero en función de una fórmula, de una correspondencia de tiempos dentro de la estructura de la frase, que ni siquiera ha alcanzado todavía la categoría de lo gramaticalmente permisible. Es decir, se trata de justificar y explicar históricamente un heclio de sincronía lingüística.

Nañez limita sus notas a poner de manifiesto la frecuencia de la fórmula simétrica 'si tuviera, diera' en Cervantes. En vista de su abundantísima documentación, piensa que este tipo de estructura sintáctica puede considerarse como un rasgo estilístico de este autor.

Además de estos estudios, pero ya no con carácter monográfico, sino formando parte de obras de carácter más amplio o general, se han dedicado capítulos de importante significación al estudio de las oraciones condicionales en español en una serie de libros cuyos autores y títulos irán apareciendo a lo largo de nuestro trabajo. Precisamente serán estos capítulos y, de los trabajos citados, los de Gessner y Kany, casi los únicos que han de sernos útiles en la segunda parte de esta investiga-

1 Garcfa diE Diego. La uniformación, p. 96.

- 2 Garcia de Uirgo, loc. cil., p. ro5. 
ción. También utilizaremos, en el mismo lugar, parte del material recogido por Cuervo en su Diccionario y por Cejador en La lengua de Cervantes ${ }^{1}$.

Nuestro estudio constará de dos partes: una, la primera, de tipo teórico-sincrónico, y otra, la segunda, de enfoque exclusivamente histórico. $\mathrm{Y}$ va a versar sobre uno de los elementos morfosintácticos de la expresión de la condicionalidad: las conjunciones o locuciones conjuntivas empleadas en español como signos de condición. Nos ha llevado a este acotamiento del campo de nuestra investigación el hecho de que todos los trabajos o consideraciones pasajeras sobre la estructura del periodo hipotético condicional en español han tenido como principal objeto de estudio la clasificación de este tipo de oraciones, su significación en función de los modos verbales, la correlación temporal entre cada uno de los niembros, es decir, entre el condicionante y el condicionado y su significación en relación con la misná ${ }^{2}$, y el uso de la conjunción si como el elemento morfosintáctico de relación más característico en la expresión de la condición en la lengua latina y en las románicas.

1 Rutino J. Cuenvo. Diccionario de construcción y régimen de la lengua castellana, 1. Paris, 1886; II, Paris, 1893; JUL,Io CEJADOR Y PRAUCA, La lengua de Cervantes (Gramática y Diccionario de la lengua castellana en El ingenioso hidalgo Don Qiujote de la Mancha"), t. I, Granditica, Madrid, 1905.

2 Nos parece muy acertada la observación de Lidia Contreras sobre la utilización de los térnuinos condicionante y condicionado en lugar de los consagrados por el uso prótasis y apódosis, por la comnotación semántica que implican en lo que al orden de aparición en la frase se refiere. No hay necesidad de justificar con ejemplos que no siempre aparece la prótasis como primer miembro en la expresión condicional. Sin enubargo, prótasis ya no se piensa, en la terminología gramatical, como el miembro que en la dependencia sintagmática condicional hace su aparición en primer término, sino como sinónimo de oración subordinada 0,10 que es lo mismo, elemento condicionante: "El periodo condicional o hipotético consta de dos partes $u$ oraciones: la subordinada, llamada hipótesis, condicion, y más comúnmente prótasis; y la principal, que recibe el nombre de apódosis". SAAIUEI, GIII X G.AYA, Curso superior de sintaxis española. Barcelona, 1955. p. 293. El térnino prótasis también aparece definido en su sentido etimológico y en el de sinónimo de subordinada de la expresión condicional: «Primera parte de una oración simple, o primera oración de una compuesta, cuyo sentido queda incompleto y pendiente de ser completado por la segunda parte de la simple o la segunda oración de la compuesta, llamada apodosis. Poco a poco (prótasis) se va lejos (apódosis); Lo que tienes, no es grave. El término se aplica principalmente a la subordinada condicionalm. FrrRnando Laszaro Carrerer, Diccionario de téminos filologicos, 2.a ed., Madrid, 1962. 
lis verdad que los trabajos de Gessner y Lidia Contreras, la Gramática de la RAE y la Grammaire des langues romanes de Meyer-Lübke, las obras de Keniston o de Kany, respectivanente ${ }^{1}$, entre otras, dedican atención a la construcción de oraciones condicionales introducidas por otras conjunciones o expresiones conjuntivas que no sean si o en que $s i$ no entre a formar parte de ellas. Pero no es menos verdad que, tanto en unos como en otras -en los trabajos especificamente dedicados al estudio de las oraciones condicionales en español o en las obras cuyo estudio está integrado en un plan más vasto-, la investigación histórica y funcional de los indicadores de la condicionalidad, independientemente de la estructura total - significativa y formal- del periodo condicional, es como un apéndice y, en todo caso, es algo incompleto, adenás cle estar planteado unas veces desde el punto de vista diacrónico, y otras, desde el sincrónico, e incluso desde el sincrónico-normativo.

Nuestro trabajo, que pretende ser cuantitativamente completo, aunque no exhaustivo, constará en su segunda parte de tres apartados: I) Conjunciones o expresiones conjuntivas que, para nosotros, tienen un valor puramente histórico; II) Conjunciones o expresiones conjuntivas que, además de poder ser documentadas, algunas, en nucstros monumentos literarios, todavia hoy tienen vigencia; III) Conjunciones o cxpresiones conjuntivas cuyo uso y documentación podemos considerar moderno, reciente.

Con este planteamiento, y con el estudio detallado de la significación de cada uno de los elementos de relación que aparezcan con valor conjuntivo, tendremos una visión teórica e histórica de conjunto del problema lingüístico que nos ocupa.

Ahora bien, antes de entrar en la casuística de los ejemplos y en el análisis de los mismos, y a pesar de las limitaciones que nos hemos impuesto en la extensión de nuestro campo de estudio, creemos necesarias algunas consideraciones generales sobre la expresión de la condicionalidad en latín en relación con las lenguas romances.

Es sabido que el elemento de relación más frecuentemente utilizado en latín para unir la oración subordinada, condicional, a la principal, condicionada, es la conjunción si, que se ha conservado en todas las lenguas románicas; bajo la forma se en italiano, francés antiguo, rumano antiguo y portugués; bajo la forma si en catalán, español, provenzal

1 MEXER-LüBKE. Grammaire des langues romanes, III, Paris, I900; RrAT. ACADEmia Españora. Gramática de la lengua española, Madrid, 1931; Chiaries I:. KANY, American-Spanish Syntax. Chicago, 195I; HAYWARD KenISTON. The Syntax of Castilian Prose. Chicago, 1937. 
y francés moderno; bajo la forma să en rumano desde el siglo xvir ${ }^{1}$. Pero las posibilidades para introducir la expresión de la condicionalidad no terminaban ahi; posibilidades que las lenguas románicas han multiplicado. Puede decirse que las construcciones básicas romances de las oraciones condicionales son las mismas que tenfa el latin, aunque ni siquiera el latín literario cumpliese a rajatabla con los esquemas gramaticales y aunque algunas de las fórmulas de las lenguas neolatinas se acuñaran en el bajo latín: "Ce schema, que le latin littéraire lui-même est loin d'avoir observé rigoureusement, a été d'autant moins maintenu par la langue de tous les jours. Le bas latin laisse entrevoir certaines tendances qui annoncent la syntaxe romane" ${ }^{2}$. Pero de la misma manera que el romance ha superado al latín en medios de expresión de los matices que se encuentran a caballo entre lo condicional y lo concesivo (uentre ces deux classes [condicional y concesiva] s'en place une troisième, celle où la réalisation de la proposition partielle est seulement possible, vraisemblable, conditionnelle, classe qui en réalité, pour la forme aussi, se trouve cn étroite comnexion avec les propositions conditionnelles: 'si mìne il vient, je m'en vais'. Ici le roman présente, dans ses moyens d'expression, une diversité beaucoup plus grande que le latinn ${ }^{3}$, también lo ha superado en el número de nexos relacionantes que puedan establecer las dependencias sintagmáticas en la expresión de la condicionalidad. Pero no sólo es que lo han superado en el número de elementos de relación condicionales, sino que incluso la estructura externa del período condicional romance, a causa de las peculiaridades de cada uno de ellos, ha conseguido borrar la imagen del periodo hipotético condicional latino: "Die hypothetische Periode im Romanischen beruht zwar auf der lateinischen, hat aber einige besondere, allen romanischen Idiomen gemeinsame $Z$ üge aufgenommen, die sie von dem lateinischen Urbilde unterscheiden. Dann haben wieder die einzelnen romanischen Sprachen innerhalb des ihnen Gemeinsamen ihre speziellen Eigentümlichkeiten ausgebildet, welche mitunter geeignet waren, das Gepräge der lateinischen Periode ihrer äusseren Gestalt fast bis zur Unkenntlichkeit zu verwischen" ". En resumen, que si, a la falta de correspondencia en el empleo de los modos $\mathrm{y}$ de los tiempos que presentan las leguas románicas respecto del latín en este tipo de oraciones, añadimos la mayor abundancia de conjunciones o de otros elementos de relación que funcionan como ta-

1 MLEYER-LÜBKE, I, pp. 539-540, y III, p. 720.

2 VEIKKO VAANANEN. Introduction au latin vulgaire. Paris, 1963, p. 177.

3 MIEYER-LÜBKE, III, pp. 719-720.

- I. GlissNER, pp. 2I-22. 
les, habremos reunido todas las peculiaridades necesarias y suficientes, creemos, que llevaron a decir a Gessner y, por lo tanto, sus palabras se hallan más que justificadas, que wwelche mitunter geeignet waren, das Gepräge der lateinischen Periode in ihrer äusseren Gestalt fast bis zur Unkenntlichkeit zu verwischen" ${ }^{1}$. Piénsese, por ejemplo, que las condiciones irreales en francés construidas con si utilizan, por modo, el indicativo; por tiempo, el imperfecto o el pluscuamperfecto, según que la proposición condicional irreal exprese un hecho presente o pasado que se vea como contrario a la realidad, o que la condición se refiera al pasado: Madame, je suis peintre (...). Si j'ETAIT sculpteur, je me PLAINDRAIS (Maupass., Fort comme la Mort, I,2); S'il [Annibal] a perdı Zama, ce n'est pas sa faute. Il l'AURAIT GAGNE, s'il AVAIT EU le soleil à dos (E. Fromentin, Dominique, III) ${ }^{2}$.

Por otra parte, la modalidad de la oración condicional en las lenguas románicas no depende de la naturaleza de la conjunción, sino de la conjugación de las categorfas gramaticales modo y tiempo: si viene, me voy o me iré; si muriera pronto, ¿qué sería de ti? -s'il vient, je m'cn irai; si je mourais bientôt, que dcviendrais-tu?" "C'est la forme clu verbe qui cxprime ici la modalité de la proposition conditionnclle» ${ }^{3}$. Y la forma verbal no depende en absoluto del elemento de relación que une las dos oraciones, sino todo lo contrario: es la forma verbal la que condiciona el empleo de tal o cual elemento conjuntivo. Una cosa es que una proposición sea condicional y otra es la modalidad de esa condición. Que es necesaria la presencia de una conjunción o de otra categoría funcional que desempeñe la misma función sintáctica para evitar anfibologías, es evidente. En 'tengo, doy' nos encontramos ante el problema de precisar la clase de dependencia sintagmática establecida. No cabe la menor duda de que estas dos formas verbales pueden estar en relación de condicionalidad ('si tengo, doy'), de temporalidad ('cuando tengo, doy') y de

1 Respecto de la concordancia y discordancia modal y temporal, y de las conjunciones y locuciones latinas que pueden introducir una oración condicional, véase ALFRED ERNoux et Françors ThOMIs, Syntaxe latine, $2^{\mathrm{e}}$ édition, Paris, 1959. pp. 374-386 y especialmente, para las conjunciones y locuciones conjuntivas (modo, dum, dummodo, ut plura non dicain, ut nilhil aliud dicam, ut ita dicam, ne, ne dicam, absque me foret), las pp. 39r-392. Se encontrará un estudio muy detenido de los distintos valores de las conjunciones que introducen una oración subordiuada en IEUMANN-HOFMANN-SZANTYR, Lateinische Grammatik, II. München, MCMLXV, pp. 572-68I.

2 Maurici: Grivisse, Le bon usage, 8e édition, Patis, 1964, pp. 1079-80.

3 Warmion v. Wartburg, Paul ZumTior, Precis de syntaxe du français contemporain, $2^{\mathrm{c}}$ édition, Bern, r947, p. III. 
causalidad ('porque tengo, doy'), determinada precisamente por los elementos relacionantes. Es decir, el elemento de relación determina la clase pero no la modalidad: 'si tengo, doy' es una oración condicional por el elemento de relación si, pero es real por las categorias gramaticales de modo y tiempo. Lo que ocurre es que determinadas conjunciones - locuciones conjuntivas sólo pueden utilizarse cuando la forma verbal se encuentra, por ejemplo, en subjuntivo: con tal de que sea eficiente, lo admitirennos; a menos que sea tonto, se dard cuenta de su situación, etc. Otras pueden emplearse tanto si la forma personal del verbo va en indicativo como en subjuntivo. Otras, por fin, según que la forma verbal vaya en indicativo o en subjuntivo introducirán una oración de tipo distinto: conno te portes bien, te haré uni regalo; como te portas bien, te hago uns regalo.

Otro aspecto interesante de la función y significarlo del elemento de relación condicional - 110 tratado en español, que sepamos ${ }^{1-}$ es el rasgo o matiz que éste puede introducir en la presentación de la condición. Ahora ya no se trata solamente de ver la simple formulación de una hipótesis condicional, sino de descubrir la matización de la hipótesis que la locución conjuncional lleva consigo. In la proposición si vinicras a casa a recogerme, le acompañaria podríamos decir que la condición ha sido formulada de una manera neutra. Pero si decimos: admitiendo que vinieras a casa a recogerme, te acompañaria, o en caso de que vinieras a casa a recogerme, te acompañaria, etc., introducimos, en la manera de plantear la condición, unas precisiones que hacen referencia al mayor o menor grado de probabilidad de que la condición se realice, a la limitación de la condición, a lo insólito de la misma, etc. En la segunda formulación -admiticndo que vinieras a casa a recogerme, te acompañaria- aludimos a un hecho cuya realización es bastante improbable; en la tercera -en caso de que vinieras a casa a recogerme, te acompañaria- la acción de la oración principal o condicionada sólo se produciría después de producirse el hecho supuesto en la subordinada o condicionante.

Cuando, incluso cuando, a un cuando, cuyo valor es, al mismo tiempo, concesivo y condicional, presentan con mayor intensidad el aspecto hipotético de la condición, es decir, del hecho que condiciona: aun cuando lo declararan inocente, seguiriamos pensando que

1 El estudio de la simtaxis del francés contemporáneo de WALTurar v. WARTbURg, Paur Zuatror. Précis de syntaxe du française contemporain, pp. II3-II7, recoge $y$ analiza algunas locuciones conjuntivas. También MAURICE GrevissE. Le bon usage, pp. 1084-1086. 
era el culpable; incluso cuando llegara a sentirse enfermo de verdad, creerian que fingia.

En las lncuciones conjuntivas a condición de que, a condición que, bajo la condición que, etc., lo que se expresa no es propiamente una hipótesis o suposición, sino una auténtica condición, en el sentido de 'calidad o circunstancia en que se promete una cosa': solamente trabajaria a condición de que me pagasen lo estipulado.

La simple suposición se expresa con las locuciones $\mathrm{s}$ u p o n i e n d o que, supuesto que, supuesto caso que: supuesto que ya no vendrán, podriamos marcharnos. Sin embargo, la locución suponiendo que puede expresar bastante más de la mera suposición: por ejemplo, la improbabilidad de la acción condicionante, $y$, en ese caso, algunas reces aparece reforzado el sentido de la expresión conjuntiva por una oración incidental de relativo: suponiendo que llegues a tiempo, lo que es mucho suponcr, no conseguirás que nadie te considere un hombre formal. Iis decir, admilicndo que y suponiendo que pueden llegar a introducir en la oración condicional el nismo matiz de improbabilidacl.

Con tal que, con tal de que no siempre tienen valor condicional, o mejor, no siempre establecen relaciones de condicionante a condicionado. Como locución conjuntiva condicional, presenta lo que se manifiesta en la condicionante como la exigencia mínima que se propone para que pueda darse lo que se expresa en la principal: con tal de que sepa estar, lo llevaremos a la rennion; con tal que sepa hacer algo, lo admitirán en la fábrica. Otras veces puede tener valor final: me sacrificaré, con tal de que se cure. Coincide en este caso con el valor de la locución por tal de que: por tal de que se calle, le daré lo que me pide. También sucle utilizarse frecuentemente en oraciones independientes de valor desiclerativo: ¡Con tal de que me ame! Aquí, el deseo está expresado con más vehemencia, cobra unos perfiles más acusados que se dijéramos: ¡Si me amara! En la primera se considera necesario que se cumpla lo deseado, aunque pueda ser dudoso; en la segunda se señala la posibilidad de conseguir la realización de lo que se desea, incluso por casualidad.

En por poco que, a poco que la restricción es más fuerte que en la locución anterior con tal (de) que. La condición es tan pequeña, aunque sea necesario que se dé para que se realice lo expresado en la oración condicionada, que se nos aparece como desproporcionada la relación entre la causa y el efecto: por poco que te equivocases, seria suficiente para que te rechazaran; por poca suerte que tuvieras, alcanzarias el triunfo.

A me nos que nos presenta la condición como algo fuera de lo 
normal, excepcional, pero no nos indica nada acerca de su grado de probabilidad: a menos que sacara fuerzas de flaqueza, no podria alcanzar la meta el primero; seguird encanallándose, a menos que se dé un milagro. Locuciones de idéntico valor o muy próximo son: excepto que, salvo que, entre las que no integran el elemento si.

Las locuciones restrictivas basta que, basta con que, sólo con que, con sólo que, con que, sóloque aluden al hecho suficiente y necesario para que lo enunciado en la principal se cumpla: con que me lo pidiera, se lo concederia; basta con que fuera anigo tuyo, atraeria las sospechas de todos. El matiz diferenciador respecto de las locuciones por poco que, a poco que, es claro.

Dado que, puesto caso que -expresión esta última poco utilizada hoy, a no ser en la lengua literaria-, s e $n$ t a do q u e presentan la condición como realizada, conceden que se ha cumplido: dado que estuviera aqui, tampoco haria nada por redimirnos; puesto caso que tuvvicra dintero, obraria de la mismia manera, lo que equivale a decir: puestos en cl caso de que tuvicra dincro, obraria de la misma mancra. Lis evidente que en estas locucioues el matiz condicional y el concesivo andan fintinamente fundidos. No podemos afirmar categóricamente el matiz dominante en cada caso, aunque tan hipotético es el uno como el otro.

La primera particularidad que hay que poner de relieve en las locuciones de otra manera, de otra suerte, otramente es que sustituyen el condicionante, pero indicando que, de no darse las circunstancias supuestas en la condición, no tendría lugar lo que se dice ell la oración condicionada: de otra manera, ni el pueblo ni el gobierno estarian nunca de acuerdo; de otra suerte, sería imposible lograr lo que te propones. Ia condición, o lo condicionante, puede ser toda una serie de razones, circunstancias, necesidades contextuales que quedan aludidas en estas expresiones.

D.e no, donde, donde no desempeñan la misma función que las locuciones anteriormente examiniadas. Los dos elementos de la primera locución suelen aparecer fundidos en el español de América. En la segunda parte de este trabajo diremos algo sobre su localización en el tiempo y en el espacio.

Siempre que, siempre y cuando, cada y cuando que, cada que no expresan tanto una hipótesis, cuanto el ineludible cumplimiento de la condición, para que se realice o no lo que se expone en la oración principal: iria al cine, siempre y cuando tuvicra dinero; siempre que no lo hicieras, ni siquiera podrias aspirar a la candidatıra. Cada y culando que es una locución inusitada hoy, incluso en la lengua literaria. En la misma situación se encuentra cada que. 
De las locuciones a cambio de que, a trueco de que, en trueco de que, a trueque de que, en trueque de que, la primera es la única que tiene vigencia tanto en la lengua coloquial como en la literaria. A trueque de que hoy sólo tiene sabor literario.

Istas locuciones presentan la acción expresada en la oración principal, en caso de cumplirse la condición señalada en la subordinada, como una transacción en la que lo que se da puede ser proporcionado o desproporcionado respecto de lo que se obtiene: a cambio de que guardaras silencio, ganarias la libertad; a cambio de ser honrado, te pagarian con el desprecio.

En 1a hipótesis de que, en caso de que, en caso que, en el caso de que, en el caso que, supuesto caso de que, supuesto caso que, etc., presentan la condición como algo cuya realización es eventual: en caso de que llegara, tendriais que avisarme; en la hipótesis de que esto sea cierto. no habrá modo de seguir adclante.

Iin estos catorce grupos de locuciones conjuntivas - solamente cil dos se analiza una sola conjunción o locución en cada uno- creemos que están recogidas prácticamente todas aquellas cuya utilización en la lengua de hoy es normal. Muy pocas: donde, denó, cada que, cada y cuando que, y alguna más, han sido agrupadas con otras locuciones actuales cuya significación es idéntica. Todos los ejemplos -que no han sido sacados de los textos- responden a las características de la lengua coloquial o escrita de hoy. Otras locuciones que tampoco cuentan entre sus formantes con la conjunción si, aparecerán en el análisis diacrónico de las mismas, que constituye la segunda parte de nuestro estudio.

Si se hiciera un balance detenido de las conjunciones o giros conjuntivos latinos que introducian una proposición condicional y que no han pasado a las lenguas romances, y de las conjunciones y locuciones conjuntivas romances de nueva creación, el resultado sería completamente favorable a las creaciones neolatinas. Pero, con ser interesante este aspecto del problema, no es el que ahora ocupa nuestra atención.

Nos interesa, por el contrario, siguiendo el esquema enunciado más arriba, ver la vida - la historia - de algunas conjunciones y locuciones condicionales. No pretendemos mostrar aquí cuándo y dónde surge por vez primera un nuevo elemento conjuncional. Pretendemos - sólo- trazar, a grandes rasgos, un esquema histórico, con referencia al momento 
actual: I) Elementos conjuntivos de puro valor histórico; II) elementos conjuntivos utilizados en otras centurias y modernamente, y III) elementos conjuntivos modernos.

\section{Elementos conjuntivos de puro valor histórico}

En este primer apartado estudiaremos las conjunciones y locuciones condicionales siguientes: adonde, donde; a tal que; a trueco de, a trueque de; cada que, cada y cuando que; como que; con que; con sólo que, sólo con que; cuando no; con tal condición que; de no (denó); de que; en tal que; otramente; por tal que; puesto que; que; se; sin; sólo que.

A d o nde, d o n de. No creemos que sea fácil explicar cómo del sentido locativo de estos adverbios ha podido nacer el de condicionalidad. Ni la RAE, ni Cuervo, ni Gessner, ni Meyer-Lübke, ni Cejador, ni Garcia de Diego dan explicación del cambio. Se limitan a constatar el uso. Tampoco añaden nada Keniston y Kany, Sintax. Gessner nos dice que ulie iiltere Sprache zicht auch das Ortsadverb donde für den Konditionalem Satz heran. Das Altfranzosische kennt diesen Gebrauch von oi gleichfalls in bescheidenem Massen (p. 58). Meyer-Lübke dice algo más que Gessner en este sentido: "Du sens locatif aussi peut dériver celui de condition" (III § 646, p. 722). Pero ¿cómo? Creemos que se podría explicar así: presentar una condición es limitar de alguna manera la acción, el pensanuiento, el deseo, etc. Cumplirla es 'situarse' dentro del marco que la condición delimita. Ahora bien, esta delimitación no sólo es de orden espacial, sino también de orden temporal; de ahí que los adverbios donde y cuando se utilizaran con valor de conjunciones condicionales.

Los ejemplos más antiguos que se recogen no van más allá de la segunda mitad del siglo xv: donde esto no hiciésenos, cayéramos en mal caso nos e todos los otros Grandes de vuestros Regnos que vuestro servicio derechamente amamos (Crónica de Don Juan Segundo, p. 549 b); do la mujer olvidares, clla te olvidará; do estas tres guardares, no es tu obra vana (Roiz $663 d$ y $447 d$, Gessner, p. 58); donde no sea esto, se podria mal esperar minguin buen suceso (Bernardino de Mendoza, Correspondencia, 338); e donde este faltasse o si assi no fuese, digo que por razon mas obligado serias (G. de.Alf, I, 85, 2I); ¿uo os pareceria mal adonde lo viessedes escrito? (Dial. de la lengua, 358, 35, Keniston, p. 400). No está muy claro que este último ejemplo tenga un decidido matiz condicional.

Donde $n o$ equivale a de lo contrario, si no, etc.: donde no, mas vale que pene el amo, que no que peligre el mozo (La Celestina, III); sin verlo, 
lo habeis de creer, confesar, afirmar, jurar y defender; donde no, conmigo sois en batalla, gente descomunal y soberbia (Quij. I, 4); que yo le dexaré libre y desembarazado: donde no, aqui morirás traydor (Quij. II, 60); que si esto El hace sin quitarme la vida, yo volveré a mejor discurso mis pensamientos; dondc no, no hay sino rogarle que absolutamente tenga misericordia de mi alma (Quij. I, 27). Algunos de estos ejemplos se encuentran repetidos en RAE, p. 394; Cuervo, Dicc. s. v. donde; MeyerLübke, $\S 646$, p. 722 , y Cejador, $\S 262$, 3. Por último: dentro de treinta dias habeis de dar caballeros que os defiendan; donde no, se procederá contra vos conforme a la ley (Guerras civ., Gessner, p. 60).

Es prácticamente imposible encontrar en los primeros siglos de nuestra literatura - siglos XII, XIII, XIV - algunas oraciones condicionales introducidas por donde. Nuestras pesquisas, si de momento no son demasiado extensas, sí lo suficientes como para poder afirmar este hecho. García de Diego se limita a decir: "De sentido locativo, la fórmula donde no, tan frecuente en los clásicos" ${ }^{1}$.

A ta 1 qu $\mathrm{c}$. Eista locución conjuntiva no aparece resgistrada ni siquiera en R RI\%, Cucrvo, Cejador y Keniston. Nosutros sólo la hemos documentado una vez, pero hay razones para pensar que, aunque no tan frecuente como por tal de que, por tal que, por tal de, debió ser bastante utilizada, por lo menos en los siglos xvi y xvir. La razón principal es que las locuciones conjuntivas condicionales encabezadas por $a$ eran frecuentísimas. Hasta tal punto que $a+$ inf. era más usual que $d e+$ inf. en nuestra Idad de Oro: "I concluded, therefore, with apparently sufficient proof, that conditional de + inf. is conserably more common today than conditional $a+$ inf. (although, the opposite was true in the Golden Age)" ${ }^{2}$. He aqui el ejemplo: por medio deste hizo a Beltrán Claquin una gran promesa de villas $y$ castillos $y$ de doscientas mil doblas, a tal que, dejado a Don Enrique, le favoreciere y le pusiese en salvo (Juan de Mariana, Historia de España, libro XVII, cap. XIII).

A trueco de, a trueque de. Fórmulas de condicionalidad muy utilizadas durante los siglos xvI y xvir. Su uso decayó totalmente en el siglo xIx: a trueco de decir una malicia, se pondrán a peligro que los destierren (Quij. II, I6, Cejador, $\S 262$, I2); a trueco de rescatarla, empeñará un hombre cuanto tiene (Dicc. Aut. s. v. trueco).

$\mathrm{Cada} q u e, c a d a$ y cuando que. En estas dos locuciones están íntimamente fundidos el matiz temporal y el condicional.

1 VICENTE Garcí DE Dirgo. Gramática listórica española, 2. edición, Madrid, I96r, p. $4 \mathrm{I} 3$.

2 KaNY, De + inf., p. I65. 
La Academia en su Diccionario de Autoridades las interpreta como equivalentes a 'siempre' y 'siempre que' o 'luego que', respectivamente: mayoria ha el Papa sobre los otros Perlados en poder e en fecho, ca ell los puede deponer cada que ficicren porque, e despues tornarlos; que la milicia que seguian por su voluntad, la podian dexar cada y cuando que quisiessen (Dicc. Aut.s. v. cada). Aliora bien, las locuciones 'siempre que', 'siempre y cuando que' modernamente tienen valor condicional. Para la primera, véase RAE, p. 394.

Cejador $(\S 262,7)$ interpreta la segunda locución como condicional: le hallaré y le desafiaré, y le mataré cada y cuando que se escusare de cumplir la prometida palabra (Quij. II, 52). Son dos locuciones cuya utilización se limita a los siglos XVI y XVII casi exclusivamente.

Como que. Esta fórmula fue muy frecuente siempre, y fue utilizada, por lo menos, hasta el siglo xvur. Participa del doble matiz comparativo y condicional: Af̧or en mano leuaua, commo que yua a caçar; alzó la espada como que lo quería ferir (Gessuer, p. 57); engañó a la Lozana, conus que fura yo Santa Ncfixa (Keniston, p. 401).

Con qu e. Iocución condicional frecuente hasta el siglo xvur con el valor de 'con condición que'. Muy utilizada, sobre todo, en la lengua forense: Mandannos que de aqui adelante... no se pueda llevar por el porte de cada arroba de las que fueren en carro o en cualesquier bestias de carga más que à razón de tres maravedís por cada legua, y à razón de un real por cada tres leguas de cada persona que fuere en los dichos carros - bestias de carga: con que esto nu se entienda en las criaturas que llevaren à sus pechos sus Madres (Dicc. Aut. s. v. con). Esta expresión conjuntiva aparece, sobre todo, en nuestros clásicos, particularmente en Cervantes, Quevedo, Mateo Alemán, etc.: Licito es al poeta escribir contra la envidia $y$ decir en sus versos mal de los envidiosos y asi de los otros vicios, con que 120 señale persona alguna (Quij. II, I6, Cejador, § 262, 5; Meyer-Lübke, $\S$ 647, p. 722); El me dió la palabra, con que no se tardase mucho (Santa Teresa, Fund., 3, Cuervo, Dicc. s. v. con); linelga de otorgar la palabra con que su alteza le cnbie la obediencia (Cisneros, Keniston, p. 402).

Con sólo que, sólo con que. De estas dos locuciones la que utilizamos hoy es la segunda. La primera sólo tiene valor histórico: yo te perdono la ofensa que me has hecho, con sólo que me pronietas y jures que... la cubrirás con perpetuo silencio, sin decirla a nadie (RAE, p. 394; el mismo ejemplo en Cuervo, Dicc. s. v. con y Meyer-Lübke, III, § 647. página 722). El ejemplo es de Cervantes, La fuerza de la sangre. Lidia Contreras cree, por el contrario, que la primera expresión citada es. "de uso modernon (p. roo). Como la mayoría de las locuciones conjuntivas. 
de estructura un poco compleja, se documenta abundantemente en la Edad de Oro.

Cuando no. Fórmula elíptica muy usual en los siglos xvr y Xvir. La registran Cuervo, Dicc. s. v. cuando, Gessner y Keniston: Nolificado que... le daria a su hija por mujer, cuando no, que le cortaria la cabeza (Patrañuclo, Gessner, p. 6o); cuando no, servirme ha de criado (Lope de Rueda, Keniston, pp. 42I-22); con lo cual se debia de contentar el rey mi suegro que hubiere de ser, y cuando no, la infanta me ha de querer de manera que, a pesar de su padre, me ha de admitir por señor y por esposo (Quij. I, 2I, Cuervo, Dicc. s. v. cuando).

Con ta 1 condición que. Locución conjuntiva bastante compleja. Pudiera ser que se deba al cruce de las expresiones condicionales con tal que y con condición que. Semejante abundancia de elementos en una fórmula es bastante característico de la lengua de la Edad de Oro. Keniston la documenta en el siglo xvI: me rogaba que le tuviese por amigo, con tal condición que los de Culua no entrasen en su tierra (Cortés, Cartas); que tu gozo y el tiempo e vuestras voluntades conformes fuessch... con tal condición que Dios donde agora te contentasse (Question de amor..., Keniston, p. 400).

De no (denó). Expresión condicional todavía vigente en Hispanoamérica, documentada por Cuervo (Dicc. s. v. de). Kany ( $D e+$ inf., p. Ior) y Lidia Contreras (p. 99). En España es absolutamente desconocida, aunque Kany piensa lo contrario: "Though not registered in the Academy dictionary is not unknown in Spain" (Sintax, pp. 297-99 apud Lidia Contreras). Es posible, incluso, que ni siquiera pueda ser documentada en los dos siglos de mayor proliferación de fórmulas conjuntivas condicionales, como lo fueron el XVI y el xvir. Nunca debió de pasar de la categoría de expresión coloquial, si es que se dio en España. Todos los ejemplos recogidos por Cuervo (Dicc. s. v. de II, $2 f$, p. 763) son de los siglos XVI, XVII y XVIII, pero no hay ni uno que no lleve el infinitivo expreso.

Junto a esta conjunción denó (de no) hay que sobreeentender un infinitivo. "In such cases de no (sometimes written denó) is equivalent to si no or de lo contrario. This colloquial construction is exceedingly common only in the Plate region, though it is found also in Ecuador and Colombia: si la pesco la enamoro, de no me voy al cine (Salvador. Camarada, p. roI)" ${ }^{1}$. Más información sobre paises hispanoamericanos que conocen el uso de denó en Lidia Contreras.

De que. Debió de ser una fórmula muy poco frecuente, tan poco

1 KAN, De + inf., p. 166. 
como lo fuera que con valor condicional. Keniston sólo registra un ejemplo: de que Dios quiere, con todos aires llueve (p. 356).

En $t_{a} 1$ que. Es una expresión condicional cuya historia la podemos remontar hasta el siglo xIv. En el siglo xvir ya es de difícil documentación. Keniston la registra para el siglo xvr: de buena gana sufricra tus aprobios, en tal que se castigara y tuvicra remedio esta hunrosa mancra de robar (G. de Alf., Keniston, p. 4Ig).

Nuestro texto pertenece al Enxieuplo XI del Libro de Patronio: Et Don Illan dixo que bien entendie quel fazia grand tuerto, pero que esto lo consintia en tal que fuesse seguro que gelo emendaria adelante (D. Juan Manuel, Libro de Patronio, XI).

Otramente. El Diccionario de Autoridades no registra esta palabra más que como adverbio de modo. Este mismo Dicc. ya la da como anticuada: "En lo antiguo tuvo mucho uso" (s. v.). Sin embargo, nos consta su empleo en la lengua escrita, y posiblemente en la coloquial, de las personal cultas de Colombia.

Con valor de conjunción condicional está documentada para el siglo xvi: otramenti ya te oucra despedido (Jiménez de Urrea, Keniston, p. 42I).

Po t t a 1 q u e. Lìn esta locución se funden el matiz condicional y final, aunque en ningún momento puede ser difícil saber cuál es el dominante. Algo de esto ocurre con la locución por tal de (véase más adelante, II) todavía vigente en nuestra lengua coloquial y literaria.

No la hemos encontrado registrada en ninguna parte. Nuestros textos son de los siglos xill y xIv: $E$ era omne que andava much a menudo en su carro por tal que lo catassen las gentes (Alf. X, Cr. Gen. de España, I72); dexavanse despeñar a furto por los adarves a dentro, et dellos [algunos de ellos] faziense muertos por tal que los levassen ende (Alf. X, Cr. Gen. de España, I72); E la gulpeja se entiende por algunos ombres... que por tal que coman bien, mandan que les abran las puertas de algun monesterio rico, por tal que pucdan vevir con los sinples monjes; otrosi muchos ombres... sc enbeodan... o fazc[n] algunas cosas que no son de facer, por tal que no[n] scan tenidos por cscasos o por fafcr lo que fazcn otros commo ellos; por tal que yo non fuesse apartado de los otros, convieneme a ser mas mallo que quisiera. Estos tres últimos ejemplos proceden del Libro de los gatos ${ }^{1}$.

$\mathrm{P}$ ues to $\mathrm{que}$. Según Gili y Gaya upuesto que y supuesto que fueron originariamente frases absolutas con participio usadas con valor

1 El libro de los gatos, "Clásicos Hispánicos", Madrid, MCMLVIII. El primer texto pertenece al cuento XXIV, pp. 76-77; el segundo al XXXIV, p. ror, y el tercero al mismo cuento y página. 
condicional y causal" ${ }^{1}$. Cejador habla del valor concesivo de esta expresión. En la lengua de hoy puesto que sólo ha conservado el valor causal.

No creemos que sea correcta la interpretación que hace Togeby ${ }^{2}$ de esta locución al considerarla equivalente a supuesto que construido con subjuntivo: "A suponer s'attache puesto que, qui avec l'indicatif, signifie "étant donné que", avec le subjontif "supposé que": podéis cobraros volviendo a reñir, puesto que sois ambidiestro (Valle-Inclán: Farsa 56). Su objeto no fue satirizar la esencia y fondo de los libros caballerescos, puesto que aumentó su número (Azorín: Lecturas 28). Eso dije, supuesto que antes Colás y Carmina se casasen (Ayala I38)" Supuesto que sí tuvo y tiene valor condicional. Nos da la impresión de que Togeby no ha encontrado ningún texto moderno donde puesto que expresara condicionalidad.

Tampoco creemos exacta la equivalencia establecida por Lidia Contreras entre puesto que y puesto caso que. Qué duda cabe que la segunda locución conjuntiva tiene valor condicional hoy, pero no la primera. A las locuciones pucsto que y supuesto que les ha ocurrido to mismo que en catalán, como puede verse en el texto de par citado por la misma investigadora chilena (p. Ior): "Avuy en catalá barccloní no l'usem, ans preferim supposat que calcada de la locución castellana supuesto quen.

In los tres ejemplos que siguen se puede ver cómo puesto que no es intercambiable con las otras dos locuciones:

$$
\begin{aligned}
& \text { supuesto que venga, lo felicitaré } \\
& \text { puesto caso que venga, lo felicitaré } \\
& \text { puesto que venga, lo felicitaré }
\end{aligned}
$$

A Lidia Contreras le ha ocurrido lo que suponemos le debió de ocı1rrir a Togeby: «No encontramos ejemplos con valor condicional, en esta expresión" (p. IOI).

Hay que decir, por último, que la expresión puesto caso que es prácticamente inusitada en el español coloquial y literario actual. Lo que podemos encontrar como expresión equivalente, pero donde puesto conserva su valor participial, es en puesto en el caso de que venga, lo felicitaré, - puestos en el caso de que venga, lo felicitaremos.

La Gramática de la RAE no recoge puesto que y sí puesto caso que entre las "formas participiales absolutas" ya totalmente gramaticalizadas ( $\$ 435$, p. 394).

2 Gili y Gaya. Curso, § 225, p. $27^{2}$.

2 KNUD TOGEנY. Mode, aspect et temps en espagnol. Købeuhavn, 1953, p. 36. 
Los textos de Keniston responden al uso de estas expresiones con valor concesivo: la tengo por la mejor... puesto caso que las otras son muy buenas; sin que daño alguno recibiésenros, puesto que peleaba con denuedo (p. 356); no digo buclto, pudiendo dezir turbio, puesto caso que el refran diga A río buelto; E puesto que ... haya habido algunas causas por do hayais sospechado... tencd por muly cicrto que aquello 20 erá por desamor (p. 399).

Valor condicional tiene puesto caso que enl este texto de Cervantes: y puesto caso que durmiesse y $n 0$ despertasse, en vano sería mi canto, si duerme, y no despierta para oyrle este nueuo Eneas (Quij. II, 44, Cejador, $\S 263,9)$.

Entre uuestras fichas no encontramos ninguna donde puesto que aparezca con valor condicional, y sí con valor concesivo: mas puesto que entre, no quiere consolación ni consejo (La Celestina, I). Sin embargo, en este caso no nos atrevemos a rechazar del todo el valor condicional.

$Q$ u e. Esta conjunción aparece empleada con valor condicional en nuestra lengua a partir del siglo Xn, en el Poenna del Cid. La cita del Pocma que ofrece Gessner no parece que tenga que ser interpretada necesariamente como condicional: Soltaricnos la ganançia que nos diesse cl cabdal (Cid, r434). Creemos que se trata - salvo que el contexto obligara a pensar lo contrario- de una oración adjetiva, donde el que, naturalmente, sigue desempeñando al mismo tiempo la función de nexo conjuntivo ${ }^{1}$. Esste mismo verso es interpretado por Menéndez Pidal (Cid, I, p. 398) y García de Diego (Grannática, p. 4I4) de la misma manera que Gessner.

Otros ejemplos: No, dijo el caballero, mas que lo fuese, qué seria por ende? (Amadis, 16, Gessner); os quiero dar 300 ducados que volvamos; son pocos los que se duelcn dél que no sea por su interese (Alonso Enríquez, Libro de la vida y costumbres y $G$. de Alf., respectivamente, Keniston, p. 400).

Nuestro texto procede del Auto primero de La Celestina: quiça con algo me quedaré, que otro $n o$ lo sabe; con que mude el pelo malo (La Celestina, I). En ninguna de las citas es evidente el valor condicional de que, salvo en la de Amadis, pero lo mismo ocurre con otras que no recogemos aqui.

$\mathrm{Se}$. Figura en este inventario la conjunción del castellano antiguo se, sólo para poner de manifiesto su existencia como variante fonética de si, circunstancia no señalada por Meyer-Lübke (I, §6I3, p. 539 y III,

1 Gili Y Gaxa. Curso, $\$ 228$, p. 275. 
$\S 643$, p. 720), ni por Bourciez ${ }^{1}$, ni por Menéndez Pidal ${ }^{2}$, ni por Keniston. Según Corominas sólo aparece hasta el siglo xIV "sobre todo o únicamente en textos leoneses, aragoneses y riojanos" ${ }^{3}$. Ofrecemos sólo tres muestras de su uso: no farian de Achilles//tan longa ledania/se sopiessen en el/|alguna conardia; se quisieras creer/|a los prouerbios antigos/ non dieras tal poder/|a uillanos mendigos (El libro de Alexandre, $\mathrm{H}$. Mendeloff, pp. 27-28). La tercera muestra la entresacamos de nuestros materiales: ¡Ciertamente se oviese en parayso canes o aves, mucho mas desearia yo alla! (Libro de los gatos, XXXII, p. 95).

$\mathrm{S}$ in . Encontramos esta preposición desempeñando función conjuntiva en el siglo xin (General Estoria). También está documentada con la misma función en el siglo xvr (Menosprecio de corte y alabanza de aldea y Lope de Rueda): sin en las leys de Dios yo non cuydasse... esta onrra mas la quisiera yo pora mi que pora otro (64I b, I4-I7, Mendeloff, p. 52); no diga nadie, que, sin son fáciles de leer, son difficiles de cumplir (Ant. de Guevara, Menosprecio, Keniston, p. 424); uvierame pesado, sin en lugar de calzas me dicran un jubón de cien ojetes (Lope de Rueda, Keniston, p. 424).

$S$ in seguido de un sustantivo o de un pronombre tiene valor condicional desde los orígenes de la lengua hasta hoy (véase, II).

Sólo que. No puede decirse que esta fórmula esté absolutamente en desuso, pero, desđe luego, es difícil su documentación. La locución con que y la más compleja sólo con que la han desplazado prácticamente de la lengua coloquial y escrita. E1 texto más antiguo que tenemos es del siglo xIrr: solo que yo pudiese la tu mano besar toda esta coyta cuidaria sanar (Berceo, Sto. Domingo, Meyer-Lübke, III, §647, p. 723). Keniston también la registra en el siglo xvi: solo que le den el pie subirá (Ant. de Guevara, Libro áureo, Keniston, p. 402).

II. Elementos conjuntivos utilizados en otras centurias y en la lengua de hoy.

A condición que. Locución documentada en el siglo xvr: a condición que los hijos que le naciessen embiassen a educar fuera de la ciudad (El Abencerraje, Keniston, p. 399); le dio la libertad, a condición

1 Edouard Bourctez. Elements de linguistique volnane. Paris, 1946, § 394 , pp. $475-76$.

2 R. MENǴndez PIDAr. Manual de Gramática histórica española. Madrid 1949, § r30, p. 340 .

3 J. Corominas. Diccionario crttico etimologico de la lengua castellana, IV, s. v. 
que el moro le avia de dar cicrtos tributos (Hita, Guerras, Keniston, p. 356). Hoy es frecuente, aunque no nos parece tanto como a condición de que: se las ve cargar heroicamente con vicjos avcriados, calaveras impenitentes y hasta con pobres diablos, a condición de que éstos desempeñen fogosamente el papel de Don Juan y se muestren pasaderamente discretos y enérgicos (Lidia Contreras, p. 74).

$A+i n f i n$ i tivo. Según Kany (p. I65) la construcción $a+i n f$. era más frecuente en la lengua de la Edad de Oro que en la de hoy, en contra de lo que opina Keniston. Por lo que a la lengua actual respecta es difícil decidirlo, porque ambas fórmulas son frecuentisimas, pero es posible que el mayor número de casos sea de de + inf. De todas formas, el fallo sobre si una fórmula es más frecuente que otra no puede emitirse basándose sólo en la lengua literaria, de lo contrario parecería que su relativa frecuencia es la misma en todas las regiones o comarcas de los países de habla hispana. Para demostrar lo errado de esta posible conclusión, podemos afirmar que en el habla diaria y en la lengua escrita de los malagueños es bastante más frecuente la fórmula de + inf. que $a+$ inf. Pero no ocurre lo mismo en otras partes de Ispaña. Que en Málaga ocurra esto se debe a que alli, y en casi toda la Andalucía occidental, se ha conservado un rasgo sintáctico del castellano antiguo y del español clásico, que hoy llama la atención por no manifestarse en ningún otro sitio, al menos con tanta virulencia como aqui. Este rasgo sintáctico consiste en la anteposición de la preposición de en las construcciones del tipo forma personal + inf.: lo ve de venir, no lo dejan de jugar, etc. Así, pues, es lógico pensar que, por influencia de esta construcción, la fórmula condicional $d e+$ inf. es más frecuente que $a+$ inf.

$\mathrm{He}$ aqui dos ejemplos; uno medieval - siglo $\mathrm{xv}$-, y otro clásico - siglo XVI-. En el Libro de los exenplos por a. b. c. leemos: $E$ acordaron de aver disputacion solenpne sobre esta question. E cada uno estudiava con grand deligencia de buscar abtoridades e rrazones suficientes para alabar cada uno al que era devoto e que era mayor ${ }^{1}$. Y en la Historia de España de Juan de Mariana: El Rey Don Pedro, desamparado de los que le podian ayudar, $y$ sospechoso de los demás, lo que sólo le restaba, se resolvió de aventurarse, encomendarse a sus manos y ponerlo todo en el trance y riesgo de una batalla... ${ }^{2}$.

Abundante documentación de este tipo de fórmula condicional se

1 Libro de los exenplos por a. b. c., Clásicos Hispánicos», Madrid, MCMLXI, cuento 57, p. 63.

2 JUan DE Mariana. Historia de España, lib. XVII, cap. XIII, en R. MENÉNDEZ PIDAL. Antología de prosistas españoles, p. I8I. 
encuentra en Cuervo, Dicc. s. v. $a$, y Cejador, 263. Keniston la registra en el siglo xvi: a baxar otro punto, sonara Lazaro (Lazarillo, Keniston, p. 420$)$.

El texto siguiente es de la primera mitad del siglo xIx y lo hemos encontrado en un página del conde de Toreno: Mientras que llegaba el socorro, queriendo Lefebvre probar la via de la negociación, intimó el I7 que a no venir a partido pasaria a cuchillo a los habitantes cuando entrase en la ciudad ${ }^{1}$.

Cejador ( $\$ 263$ ) dice que $a+$ inf. "es giro idiomático del castellano». Se puede afirmar que, por lo menos, también lo es del francés: $A$ les détailler, les traits de madame Gance n'avaient rien d'extraordinaire (A. France, Le lib. de m. ami, p. I73, en M. Grevisse, Le bon usage, p. I086). Según Gessner, esta construcción y con este valor, es tan frecuente en español como en francés: "Sher gewöhnlich, wie im Französischen, der Infin. mit á: á no volverle la espada en el camino, aquel solo golpe fuera bastante para dar fin à su rigorosa contienda, Quij. 35" (pp. 59-60).

$\mathrm{C}$ a s o qu e. Locución ya registrada en el siglo xvr: Caso que esto sea verdad, es tan poco lo que ganamos (Antonio de Guev., Libro áureo, Keniston, p. 403). La Gramática de la RAE, ejemplifica con esta locución: caso que venga, avisame ( $\$ 435$, p. 394). Actualmente son más frecuentes en caso de que, en el caso de que, en caso que, etc.

Como. El empleo de como con valor condicional parece que no va más allá del siglo xvi: será mucha ayuda tratar con ellos, como sean virtuosos (Sta. Teresa, Vida XIII, Cuervo, s. v. como); como haya muchas truchuelas podrán servir de una trucha (Quij. I, 2, RAE, p. 293); que mi padre vendria en ello, como yo se lo dixesse (Quij. I, 24, Cejador, § 262, 2); este verano se teme que, como el turco.esté victorioso, se pondrá en hacer guerra (Cisneros, Keniston, p. 400); como usted me trueque las papeletas, le trituro (Galdós, Fort. y Jac., Meyer-Lübke, III, § 647, p. 723).

Con condición que. Locución condicional registrada hasta ahora en los siglos XVI y XVII: pero con condición que me deis vuestra palabra... e yo os diré lo que acá dél se dice (Libro de la vida... Keniston, p. 400); que les pagaria el barco de bonissima gana, con condición que le diessen libre y sin cautela a la persona (Quij. II, 29. Cejador, §262, 8).

Uno de nuestros textos pertenece al primer cuarto del siglo xv: Dizen que un ombre dio un puerco a muchos ciegos con condición que lo matassen a palos (Libro de los exenplos..., 64, p. 69).

1 EI, CONDI: DE TORENO. Historia del levantamiento, guerra y revolución de España (1835-37), en Antologia de prosislas españoles, p. 378. 
Con + infinitivo. Hay que suponer que este tipo de coustrucción con valor condicional debe ser bastante anterior al siglo xvr. Sin embargo, todos los investigadores que lo registran no ofrecen textos anteriores a este siglo: con mostrar don Antonio la caballeria de lo alto en las cras del lugar, los enemigos fueran detenidos o se retiraran (Mendoza, Guerra de Granada, III, Cuervo, s. v. con y Keniston, p. 534); con deciros tres reglas... pensaré aver cumplido con vosotros (Diálogo de la leng., Keuiston, pp. 420 y 534).

Cou sólo + infinitivo. Fórnula no registrada en Keniston ni en ningún otro investigador. Nuestro texto es del siglo xvi y pertenece a Fray Luis de León: $y$ veremos que con sólo tener los ojos enclavados en él-con atención... los descos nuestros y las afecciones turbadas... se van aquietando poco a poco (Nombres de Cristo, lib. II, cap. III).

Con ta 1 q u e. Expresión conjuntiva condicional utilizada posiblemente por vez primera en el siglo xvi. Hoy es una de las más utilizadas en la lengua coloquial junto a con que, prescindiendo, claro está, de si: que te adornes con el hábito que tu oficio requiere, con tal que sea limpio (Quij. II, 5I); con tal, que tengan carta de examen de los Caciques de los Poetas (Quev., Vida del gran tacaño, Dicc. Aut.s. v. cacique); les dijo que con tal que le asegurasen de hacerlo así, sería contenta (Quintana, Don Alvaro de Luna, RAE, p. 394 y Cuervo, Dicc. s. v. conn). También Keniston (p. 402).

Entre nuestros textos encontramos uno que ofrece la particularidad de aparecer destruida la unidad locutiva por haber intercalado el autor la conjunción adversativa empero: lo que determinasen por lo menios los seis de ellos, con tal, empero, que de cada cual de las naciones concurriese un voto, aquello ficese valedero y firme (Juan de Mariana, Hist. de España, lib. XX, cap. II). Se nos ocurre pensar que esto era posible, porque la expresión conjuntiva era sólo con tal. E1 Dicc. de Aut. define con tal como "modo adverbial, con que se previene alguna condición, o calidad en algún pacto, o tratado" (s. v. tal): En cualquier moneda vieja y mueva, con tal que corra, y se reciba por precio. Hoy que se siente como elemento solidario de los anteriores.

$\mathrm{Cu}$ a n d o. Al ocuparnos de los adverbios de lugar donde, adonde (I) expusimos la posible causa por la que este adverbio de tiempo se iria cargando de contenido condicional. Su documentación data del siglo xIrI, aunque es posible que pueda ser documentada antes: Quando non lo leycsse, decir non lo querria (Berceo, Sto. Domingo, Gessner, p. 58); Soy pecador e pleno de grant vicio, quando tu non quieres, $y$ yo bevir non cobicio (Libro de Alex., Meyer-Liübke, III, § 645, P. 72I); yo tengo ganado lo que prometi, $y$ cuando no fuere verdad $y$ se viere palpablemente, castigucnme como quisieren (G. de Alf., Cuervo, Dicc. s. v. cuando). 
Nuestro texto es del siglo xrv: $e$ despues quando se querelan del al obispo, porna y otro peor (Libro de los gatos, XVI, p. 60). Debemos indicar que no siempre es fácil decidir el matiz de la oración introducida por cuando.

Dado que. Frase conjuntiva totalmente gramaticalizada cuya documentación arranca del siglo xvr: $y$ dado que dificil se te haga... piensa que en las grandes afrentas se conocen los grandes corazones (Tragicomedia... Elicia, Keniston, p. 392); dado que sea verdad lo que dices, cuenta con mi aprobación y mi ayuda (RAE, p. 394).

$\mathrm{De}+$ infinitivo. Ya hemos hablado de esta construcción de valor condicional ( $I$, de no). Pero lo que antes ocupó preferentemente nuestra atención fue la fórnula de + negación sin inf. expreso. $D e+$ inf. es mucho más frecuente en el habla coloquial que en la lengua literaria de España; no obstante, puede decirse que va ganando terreno por días. Hay constancia de su utilización desde el siglo xvi: $Y$ de no tratarlo ansi, se queja Dios que le han ensuciado su nombre (Avila, Eucar., Cuervo, Dicc. s. v. de); de volver a mirallos, huyen (G. de Alf., I, Keniston, p. 420); En caso de ceder a un anor, ella no hubicra tenido en cuenta... (Gálvez, El cántico espiritual, p. I56, Kany, De + inf., p. I66).

De otra manera, de otra suerte. Istas dos locticiones equivalen a si no. Las dos tienen cierto sabor culto, de lengua escrita, sobre todo la segunda. Esstán documentadas ya en el siglo xvr: de otra manera muestro señor seria mucho deserujdo (Cisneros, Keniston, p. 420); de otra suerte ni tu lealtad ni mi ser lo consentirian (El Abencerraje, Keniston, p. 420 ).

En caso que. Fórmula conjuncional registrada desde el siglo xvr: en caso que el Rey de Romanos viniese a Apulla, seria inconveniente (Cartas del Gran Cap., Keniston, p. 403); en caso que el juez no haga lo que es en si hacer... cada uno le puede decir lo que Cristo dijo (Libro de la vida..., Keniston, p. 4I9).

En $t$ a 1 c a so. No poseemos documentación anterior al siglo xvr: en tal caso no seria dino de reprehension (Dial. de la leng., Keniston, p. 420).

Gerundio. El empleo del gerundio con valor condicional es frecuentísimo en la lengua coloquial española, creemos que bastante más que en la escrita. Hay verbos cuyos participios y gerundios, por la significación intrínseca del verbo, son muy utilizados con valor condicional: suponiendo, admitiendo, imaginando, etc. Los gerundios con valor condicional obedecen, generalmente, a la fórmula ger. +que. Los que no pueden construirse según esta fórmula son los de los verbos auxiliares.

Poseemos textos con gerundios de valor condicional a partir del siglo xv: Del mundo me quejo, porque no me dando vida, no engendrara en él 
á Melibea; no nascida no annara (La Celest., Gessner, p. 60). Obsérvese también el valor condicional del participio. De los siglos xVI y xvIr hay abundante documentación: mostrándonos esso exemplos, avreis cumplido... vuestra promesa (Dial. de la leng., Keniston, p. 42I); poniendo los ojos la prudencia de v. E. en mi buen desseo, fio, que no desdeñará la cortedad de tan humilde seruicio (Quij. I, I, Cejador, § 264, I).

Ofrecemos por nuestra parte un texto de la Derrota de los pedantes: Díjoles también que los hombres habian nacido para trabajar, y muy pocos entre ellos para saber; porque ciertannente aquellos pocos, siendo buenos, bastan para ilustrar a todos los demás con su sabiduria (L. F. de Moratin, Derrota, Aizt. de pros., p. 358).

Por + infinitivo. Esta fórmula sintáctica de valor condicional es casi tan antigua como nuestra lengua: Non falsaric su dicho por aver monedado (Berceo, Sto. Domingo, Gessuer, p. 59); La mujer sin verguenza por darle diez Toledos Non dexaria de facer sus antojos aredos (Roiz, Gessner, p. 59).

Por poco que. Actualmente esta locución también puede tener valor condicional junto al predominantemente concesivo que ha tenido siempre y que conserva todavía. Keniston sólo la registra con valor concesivo: Por poco que esso valga, hay para nos entregar de la deuda (Laz., Keniston, p. 399).

Por ta 1 de que. No se puede dar por totalmente anticuada esta expresión con valor condicional. Es verdad que hoy parece ser dominaute el sentido final, pero no ha enterrado del todo el condicional (Lidia Contreras, p. Ior; la única que registra la expresión siguiendo a Förster). En frases de este tipo: por tal de no oirte, te daria lo que me pides puede verse con cierta claridad el matiz condicional.

Por tal de aparece ya en el siglo xuI con perfiles semánticos poco precisos. Nuestros textos pertenecen a la Crónica General de España: E tan grand estudio ponie de guardar la voz, cuemo nos de suso dixiemos, que por tal de la guardar, cuando avie de llamar algun cavallero, otri lo llamava por el (Alf. X, Cr. Gen., I72); començo a pensar Nero en muchas guisas por tal de no aver a obedecer a Galba (Ibid., I78); a las vezes qu $e$ dixiessen exicmplos dalgunos que se mataran, por tal de avivalle el coraçon que se pudiesse el matar (Ibid., I78).

$\mathrm{Sa} 1 \mathrm{vo}$ que. Gessner interpreta las locuciones salvo que, salvo porque como sustitutos de la oración condicional («als Ersatz des konditionalen Satzesn, p. 60). Keniston registra el valor adversativo y de exceptuación de la locución salvo que, matiz este último que, evidentemente, está muy cerca de lo condicional: No hay otro que dezir salvo que la gente de pie ya ha salido (Cartas Gran Cap., Keniston, p. 356). Lo que parece 
estar fuera de duda es el valor condicional que actualmente puede tener esta expresión: salvo que vinieran a invitarme personalmente, no iria a la .rpertura de la exposición.

Los textos aducidos por Gessner son muy claros: Mucho mas te diria, salvo que no me atrevo (Roiz, 4II d); No es dubda que lo hicieran, salvo porque Fernan Alvares les esforzó mucho (Cr. de Don Juan II, 522 a);

$\mathrm{S}$ in $\mathrm{q}$ u e. Expresión documentada con valor condicional a partir del siglo xvi ('si no'): no lo dexemos sin que primero lo esaminemos (Dial. de la leng., Keniston, p. 403). Cejador ( $(265)$ y Spaulding ( $(96)^{1}$.

$\mathrm{S}$ in + sust. o pron. Construcción de valor condicional que podrá ser documentada desde los origenes de nuestra lengua. Hay documentación del siglo xvi: sin carta no creerá mi palabra (Penitencia de amor, Keniston, p. 42I).

Supuesto (caso) que. Es posible que esta locución no tuviera valor condicional en los siglos XVI y xvIr; puesto que no la registran con tal valor ni Cuervo, ni Cejador, ni Keniston. El testimonio más antiguo que tenemos es el de la RAE (p. 394): ¿Y qué le importa a usted, supucsto que tan de veras aborrece la sociedad? (Bretón, Los solitarios, esc. X). Li1 Diccionario de Autoridades - y la Gramaitica de la RAE, $\S 438$, p. 398- registra supuesto que como equivalente de puesto que y bien que (s. v. suponer). Ahora bien, puesto que "vale lo mismo que aunque») (s. v. puesto): Que él, por estar impedido de enfermedad, no lo podia hacer, pucsto que mucho lo deseaba (Mariana, Hist. de España, lib. XIV, cap. XVI); ¿Y escribisteisla vos? dixo la Duquesa: Ni por pienso, respondió Sancho, porque yo no sé leer ni escribir, puesto que sé firmar (Quij. II, 36). Es decir, sólo documenta el valor concesivo, que después fue destacado por Cejador ( $\$ 265)$. Por estas razones creemos que se puede afirmar que el valor condicional de supuesto que arranca del siglo pasado, y que puesto que nunca tuvo valor condicional (véase, I, puesto que).

Ya que. En la actualidad esta locución ha perdido prácticamente todo su sentido condicional. Si conserva algo es en estado tan precario como por tal de. La RAE (pp. 350 y 394), Gili y Gaya ( $\$ 248$, p. 296) y García de Diego (p. 4I3) registran este valor. La RAE también señala su valor concesivo (p. 398). Textos condicionales: y ya que coman, sea aquello que hallaren mais a mano (Quij., I, Io, Cejador, § 262, I0); pues sealya que no bien vista, bien/mantenida (Calderón, Duelos de amor y lealtad, III, I7, RAE, p. 394); ya que tu desgracia no tiene remedio, llévala con paciencia (RAE, p. 394).

1 Robert SpauldiNg. Syntax of Spanish Verb. New York, 1952. 
III. Elementos conjuntivos modernos.

Nos vamos a limitar en este tercer apartado, puesto que la documentación es innecesaria, a presentar en orden alfabético los giros conjuntivos que consideramos modernos, es decir, no documentados antes del siglo xx: a condición de que, admitiendo que, a menos que, a poco que, bajo condición de que, bajo condición que, basta con que, basta que, con + inf.. con la condicion de que, con tal de que, desde que (Hispanoamérica), en el caso de que, en la hipótesis (de) que, en la suposición (de) que, es cuestion de que, la cuestion es que, llegado (de) que, siempre y cuaindo, todo consiste en que, todo esta en que, etc.

Dos finalidades principales hemos perseguido al redactar estas páginas: una, contribuir al mejor conocimiento de la función y significado de las conjunciones y locuciones conjuntivas condicionales en el español de hoy; otra, ordenar y estudiar históricamente estos mismos elementos de relación.

Como dijimos, esta ordenación y estudio no es ni exhaustiva ni completo, pero creemos que puede tomarse como un principio de clasificación orgánico e histórico. Más adelante, cuando dispongamos de un buen caudal de citas de primera mano, volveremos sobre esta segunda parte de nuestro trabajo para ampliar, rectificar o eliminar. Además, estamos casi seguros de que todavía aparecerán algunas otras nuevas fórmulas desconocidas o pasadas por alto pertenecientes a la lengua de los siglos XII al xv.

Del interés de este tipo de trabajos podrá juzgarse cuando se emprenda la tarea, $\tan$ ardua como arriesgada, de escribir la sintaxis histórica del español.

Granada.

JosÉ MONDÉ JaR. 